\title{
USO DA MICROSCOPIA DE LUZ TRANSMITIDA NA CARACTERIZAÇAO DE FLUXANTES *
}

\author{
Cássio Santos de carvalho ${ }^{1}$ \\ Anderson Pereira Martins ${ }^{2}$ \\ Letícia Miranda Alves ${ }^{3}$ \\ Hiury Voltz ${ }^{4}$ \\ Valdenir Moreira Junior ${ }^{5}$ \\ Cleidisson Barbosa Lorenzoni ${ }^{6}$ \\ Estéfano Aparecido Vieira ${ }^{7}$
}

\section{Resumo}

Os pós fluxantes consistem de uma mistura de minerais que em contato com o aço líquido, devem fundir e gerar uma escória líquida com características específicas para cada condição de lingotamento. Possuem, como base, o sistema $\mathrm{CaO}-\mathrm{SiO}_{2}-$ $\mathrm{Al}_{2} \mathrm{O}_{3}-\mathrm{Na}_{2} \mathrm{O}-\mathrm{CaF}_{2}$ e podem conter $\mathrm{MgO}, \mathrm{Li}_{2} \mathrm{O}, \mathrm{K}_{2} \mathrm{O}$ e $\mathrm{B}_{2} \mathrm{O}_{3}$. Este trabalho propõe caracterizar os pós fluxantes comerciais baixo carbono e peritético através da microscopia ótica de luz transmitida usando os mesmos procedimentos de descrição de lâminas delgadas para rochas e minerais. Os resultados mostraram a formação de grãos e fase cristalina bem definidas para o peritético e a fase amorfa para o baixo carbono. Em trabalhos futuros esta técnica melhor desenvolvida poderá servir para quantificar o nível de cristalinidade dos fluxantes com maior precisão.

Palavras-chave:Pós Fluxante; Lâmina Delgada; Lingotamento; Microscopia óptica.

\section{TRANSMITTED LIGHT MICROSCOPY USE IN FLUXING CHARACTERISATION}

\begin{abstract}
The casting powder consists of a mixture of minerals in contact with the liquid steel must merge and produce a liquid slag with specific characteristics for each casting condition. Feature based on the $\mathrm{CaO}-\mathrm{SiO}_{2}-\mathrm{Al}_{2} \mathrm{O}_{3}-\mathrm{Na}_{2} \mathrm{O}-\mathrm{CaF}_{2}$ system and may contain $\mathrm{MgO}, \mathrm{Li} 2 \mathrm{O}$, $\mathrm{K}_{2} \mathrm{O}, \mathrm{B}_{2} \mathrm{O}_{3}$. This work aims to characterize the post commercial low carbon fluxes and peritectic through the optical transmitted light microscopy using the same Description of thin sections of procedures for rocks and minerals. The results showed the formation of grains and well-defined crystalline phase to the amorphous phase and peritectic to low carbon. In future work best developed this technique may serve to quantify the level of crystallinity of the fluxes accurately.
\end{abstract}

Keywords: Flux powder; solid slag film; casting; optical microscopy.

1 Bolsista de Pós-Doutorado do Programa de mestrado em metalurgia e materiais do Instituto Federal do Espírito Santo - IFES, Vitória - ES, Brasil.

2 Técnico Metalurgia, Técnico de laboratório/ metalurgia, Instituto Federal de Espírito Santo (IFES), Vitória, ES, Brasil.

3 Engenheiros Metalurgistas e Mestrandos em Engenharia Metalúrgica e de Materiais do Programa de Pós-Graduação em Engenharia Metalúrgica e de Materiais, PROPEMM, Vitória, ES, Brasil.

4 Engenheiros Metalurgistas e Mestrandos em Engenharia Metalúrgica e de Materiais do Programa de Pós-Graduação em Engenharia Metalúrgica e de Materiais, PROPEMM, Vitória, ES, Brasil

5 Mestre em Engenharia de materiais Doutorando em engenharia de Materiais do Programa de Pós-Graduação em Engenharia e Ciência dos Materiais. Campos dos Goytacazes, Rj, Brasil.

6 Especialista em preparação de lâminas delgada. Técnico do laboratório de caracterização daFaciFaculdade Cachoeiro de Itapemirim - ES, Brasil.

7 Engenheiro Metalúrgico, Prof. Dr. Professor do Programa de Mestrado em Engenharia Metalúrgica e Materiais do Instituto Federal de Espírito Santo (IFES), Vitória, ES, Brasil. 


\section{INTRODUÇÃO}

Os pós fluxantes para lingotamento contínuo consistem em uma mistura de minerais envolvendo basicamente o sistema $\mathrm{CaO}-\mathrm{SiO}_{2}-\mathrm{Al}_{2} \mathrm{O}_{3}-\mathrm{Na}_{2} \mathrm{O}-\mathrm{CaF}_{2}$. Quando em contato com o aço líquido deverão fundir a uma temperatura em torno de $1040^{\circ} \mathrm{C}$ a $1300^{\circ} \mathrm{C} \mathrm{e}$ gerar uma escória líquida com características específicas para cada condição de lingotamento [1]. Suas funções no processo têm como objetivos principais transferir o calor do metal em processo de solidificação e garantir a lubrificação entre a parte sólida e o molde [2]. Além disso, os fluxantes atuam também como isolantes térmicos, proteção contra a oxidação e absorção de inclusões na superfície $[3,4,5]$. Todas estas propriedades terão um efeito decisivo na qualidade e na produtividade no processo de lingotamento.

A composição dos fluxantes variam de acordo com o tipo de aço a ser lingotado. $O$ fluxante baixo carbono é caracterizado por apresentar predominância de fase vítrea que facilita a transferência de calor na interface placa/molde em solidificação [2]. No caso dos fluxante peritéticos existe tendência para formação de camada cristalina que inibe a transferência de calor por radiação devido à opacidade desta fase. Portanto, um pó fluxante ao se cristalizar reduz sensivelmente o fluxo de calor da placa/molde $[3,7]$.

A petrologia é uma área da geologia que utiliza a microscopia ótica para identificação de minerais, através de lâminas delgadas e microscópico ótico de luz transmitida [8,9]. A lâmina delgada ou seção delgada consiste na colagem da rocha na lâmina de vidro com posterior polimento com pó abrasivo até a espessura desejada de $0,03 \mathrm{~mm}$. A fina camada de rocha permite a passagem de luz pelos minerais translúcidos, que através de técnicas como a polarização, ângulo de extinção, hábito, birrefringência e relevo possibilita sua identificação [12].

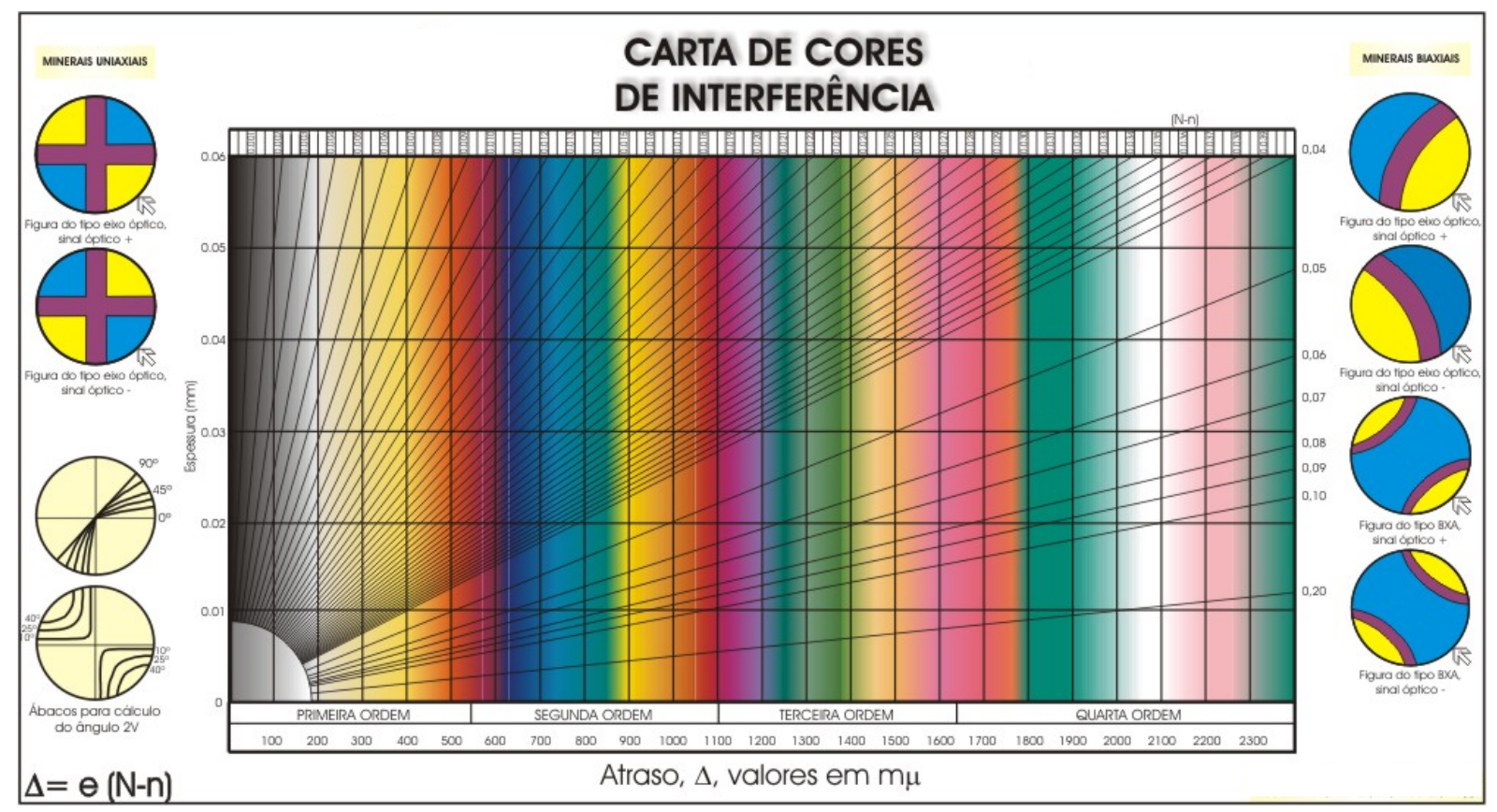

Figura 1. Carta de cores de interferência [10].

A luz emitida pelo microscópio é constituída por ondas que vibram em direções aleatórias, mas com a adição de polarizador a luz se polariza em uma direção e quando atravessa o mineral é repartida em duas direções perpendiculares entre si, permitindo identificar minerais biaxial e uniaxiais. As cores observadas ao 
microscópio com a introdução do analisador são denominadas cores de interferência. Elas variam conforme a orientação do cristal e a espessura da lâmina como mostra a Figura 1 [10]. Assim a proposta deste trabalho é caracterizar fluxantes utilizando-se esta técnica já que estes possuem características físicas muito similares aos minerais encontrados na natureza.

\section{MATERIAIS E MÉTODOS}

O presente estudo utilizou lâmina delgada para caracterização de dois pós fluxantes comerciais, um deles utilizado para aços peritéticos e outro para aços baixo carbono, cujas composições químicas estão apresentadas na Tabela 1. Os resultados foram calculados por meio de balanço de massa a partir de análises obtidas por espectroscopia por energia dispersiva (EDS). As análises foram feitas em um equipamento da Marca Oxford acoplado a um microscópio eletrônico de varredura (MEV) da Marca Zeiss Mod. Evo 10 pertencente ao Laboratório de Microscopia Eletrônica de Varredura do IFES, campus Vitória. As composições obtidas provavelmente possuem erros que podem variar de $5 \%$ a $20 \%$ especialmente para elementos em menor concentração e mais leves. No entanto, as análises mostram concentrações que estão de acordo com o esperado, pois menores concentrações de $\mathrm{F}$ e de $\mathrm{CaO}$ foram identificadas para o fluxante baixo carbono.

Tabela 1. Composição química dos fluxantes comerciais peritético e baixo carbono obtidas via balanço de massa a partir dos resultados de EDS.

\begin{tabular}{ccc}
\hline COMPOSTOS & $\begin{array}{c}\text { FLUXANTE } \\
\text { PERITÉTICO (\%) }\end{array}$ & $\begin{array}{c}\text { FLUXANTE BAIXO } \\
\text { CARBONO (\%) }\end{array}$ \\
\hline $\mathrm{CaO}$ & 26,35 & 21,56 \\
$\mathrm{SiO}_{2}$ & 26,65 & 32,76 \\
$\mathrm{Al}_{2} \mathrm{O}_{3}$ & 4,18 & 3,55 \\
$\mathrm{MgO}$ & 1,5 & 2,29 \\
$\mathrm{Na}_{2} \mathrm{O}$ & 5,55 & 10,25 \\
$\mathrm{~K}_{2} \mathrm{O}$ & 0,24 & 0,24 \\
$\mathrm{Fe}_{2} \mathrm{O}_{3}$ & 1,15 & 0,85 \\
$\mathrm{~F}$ & 12,06 & 9,09 \\
$\mathrm{CaF}_{2}$ & 0 & 0 \\
$\mathrm{CO}_{2}$ & 22,32 & 19,42 \\
$\mathrm{TOTAL}$ & 100 & 100 \\
\hline
\end{tabular}

\subsection{Preparação das amostras}

A preparação das amostras seguiu a metodologia de "Stolberg" com algumas modificações, considerando-as mais próximas dos parâmetros industriais [7]. Os fluxantes comerciais foram descarbonetados a temperatura de $650^{\circ} \mathrm{C}$ por 16 horas ao ar e então foram colocadas $30 \mathrm{~g}$ destas amostras em cadinhos de alumina para aquecimento a $1300^{\circ} \mathrm{C}$ por 20 minutos. As amostras fundidas foram despejadas em um cadinho de aço inoxidável e aquecidas até $610^{\circ} \mathrm{C}$ por 20 minutos e depois resfriadas naturalmente.

A descarbonetação é necessária para a eliminação dos compostos voláteis e consequente diminuição da evaporação dos componentes durante o aquecimento [3]. Já a temperatura escolhida de $1300^{\circ} \mathrm{C}$ visa garantir a total fusão do fluxante. Por 
fim o recozimento na temperatura de $610^{\circ} \mathrm{C}$ favorece a indução da cristalização do fluxante [11].

\subsubsection{Confecção das lâminas}

As lâminas foram confeccionadas seguindo padrões da ABNT/NBR [12,13]. Após o resfriamento, as escórias foram lixadas para nivelamento da base e posteriormente foram coladas em lâminas de vidro. Em seguida, foi feito um lixamento até que se atingisse a espessura desejada de $0,03 \mathrm{~mm}$. Esta operação pode ser simplificada em 3 etapas:

- Lixamento e colagem da amostra na lâmina;

- Corte e desbaste;

- Polimento.

\section{Lixamento}

Primerio as faces das amostras foram lixadas em politrizes, com auxílio de pasta de abrasivo e água, até ficarem completamente planas. Foi utilizado abrasivos de carbeto de silício na granulometria de 180 e 320 mesh, nivelando a base para ser colada na lâmina. Usando-se resina epóxi uniu-se a escória de fluxante fundido à uma lâmina de vidro. Durante a cologem deve-se fazer fricções laterais para eliminar todas as bolhas de ar retidas entre escoria/lâmina. Para uma colagem perfeita, as lâminas devem estar livres de impurezas e vestígios de gordura [12,13]. A Figura 2 mostra o aspecto macroscópico obtido para ambas as amostras. As amostras foram cortadas/desbastadas com auxílio de uma máquina da marca Polipan-2 Pantec $220 v$, do IFES, campus Vitória $[12,13]$.

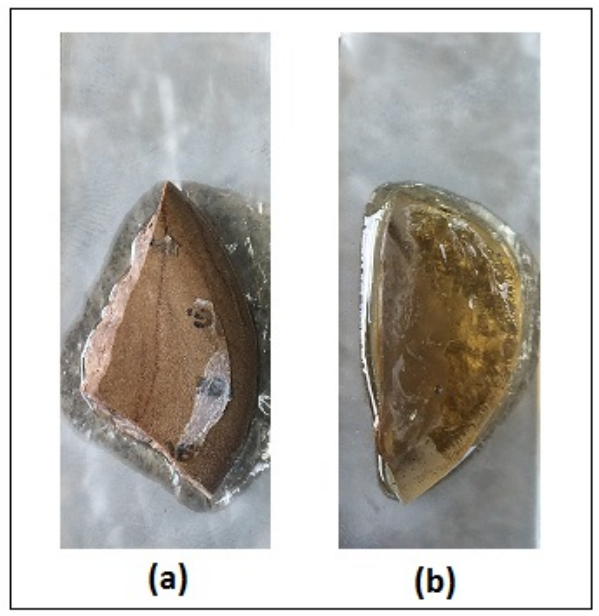

Figura 2. Amostras planificadas e coladas na lâmina, (a) fluxanteperitético e (b) fluxante baixo carbono. 


\section{Corte e Desbaste}

Nesta etapa, as amostras coladas na lâmina foram submetidas a um corte, no qual foi obtida espessura inferior a $2 \mathrm{~mm}$ e superfície paralela à lâmina [12,13]. O corte foi realizado na máquina Militon Cortadora Metalográfica de Pequena Dimensão, $220 \mathrm{~V}$, pertencente ao laboratório de Engenharia de Superfícies do IFES, campus Vitória.

\section{Polimento}

O polimento final teve como objetivo a diminuição da espessura das amostras até aproximadamente 100 micra através do uso de abrasivos de carbeto de silício, preparados sob a forma de pasta, de números 180, 320, 600 e 1700 mesh. O polimento final foi feito usando-se abrasivos de carbeto de silício de 3000 mesh, onde atingiu-se uma espessura de aproximadamente $0,03 \mathrm{~mm}$. A verificação da espessura e do paralelismo da amostra na lâmina foi feita com microscópio petrográfico, seguindo a referência da birrefringência máxima dos minerais cuja espessura correspondente encontra-se na Figura $1[12,13]$. Os polimentos foram realizados em uma máquina da marca Polipan-2 Pantec 220V, do IFES, campus Vitória.

As lâminas delgadas e polidas foram analisadas no microscópio petrográfico de luz transmitida, Leica DM750P pertencente ao IFES, campus Vitória, para análise das propriedades ópticas. Foi utilizado recursos como a polarização, rotação da platina, compensador de $1 \lambda$ e captura das imagens com ajuda do software $L a z E Z$.

\section{RESULTADOS E DISCUSSÃO}

A utilização da microscopia de luz transmitida permitiu avaliar o nível de presença de cristais nos fluxantes. Conforme será mostrado adiante, será visto que um se apresentou cristalino e o outro amorfo. A Figura $3 a$ e $3 b$ apresenta as secções polidas do fluxante baixo carbono e de um vidro respectivamente. Este último foi utilizado para efeito comparativo no qual foi evidenciado a inexistência de quaisquer propriedades ópticas em ambos materiais [14].
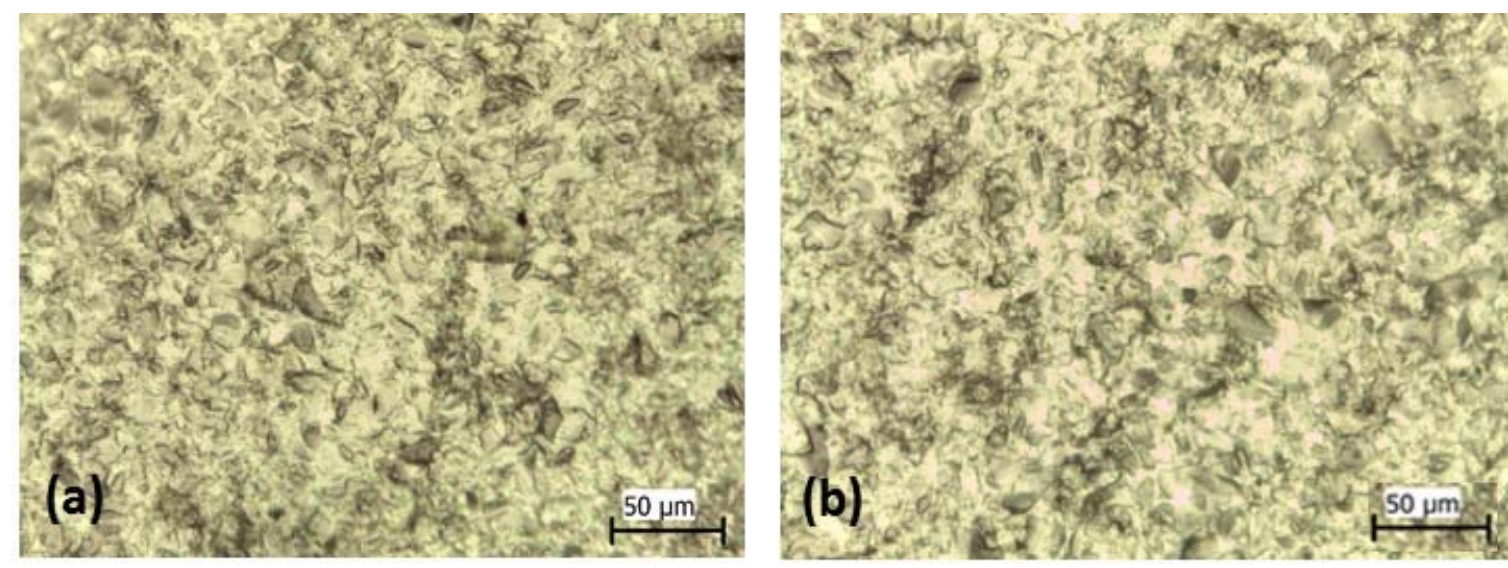

Figura 3.Lâmina delgada do pó fluxante Baixo carbono (a) e do vidro, (b). 
Nas Figura $4 a$ e $4 b$ temos a secção polida do fluxante peritético em diferentes aumentos, onde evidenciou-se a formação de grãos claramente delimitada. Estes grãos não podem ser denominados de minerais, pois acredita-se que não possuem uma composição química suficientemente uniforme, sendo portando, classificados como mineralóides [14]. Acredita-se que o tempo e a temperatura a que foram submetidos não foram suficientes para sua melhor formação, impossibilitando uma clara identificação. Mas, quando foi aplicado técnicas de identificação mineral, observou-se hábitos semelhantes a de minerais, como extinção e birrefringência apresentado na Figura 5, características típicas de minerais.
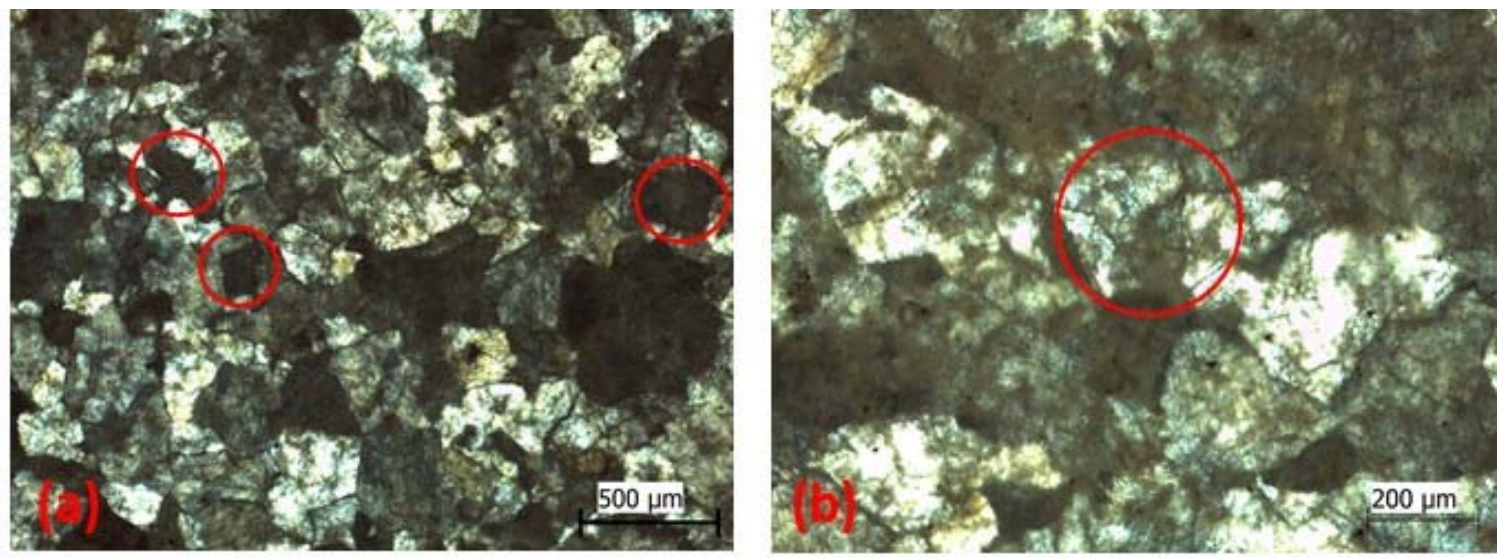

Figura 4. Escória do pó fluxante peritético em lâmina delgada. Em destaque os mineralóide de Calcita.

$\mathrm{Na}$ Figura 5 podemos ver que as secções polidas do fluxante peritérico com nicois cruzados. Em análise mais detalhada presume-se que os mineralóides formados são de $4^{a}$ ordem com birrefringência extrema de 0,17 , relevo baixo, uniaxial negativo $[14,16]$. Quando foi acrescentado o compensador, as cores passaram de pleocróicas para cores extremas de $2^{a}$ ordem, características típicas da calcita $\left(\mathrm{CaCO}_{3}\right)[15,16]$. Pôde-se confirmar a formação da calcita ao se adicionar ácido clorídrico $25 \%$ sobre a escória, onde notou-se a "fervura" do material, efeito que caracteriza a liberação do carbono para a atmosfera, característica típica dos carbonatos.
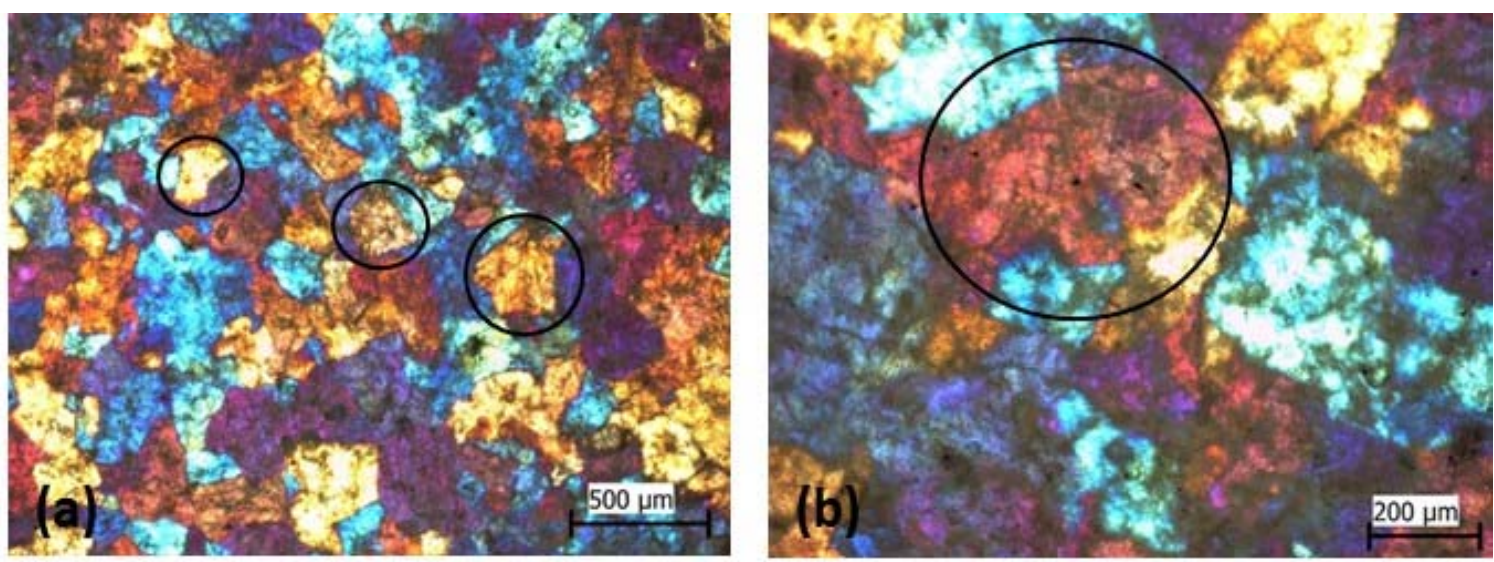

Figura 5. Escoria em lâmina delgada usando nicois cruzados e compensador de $1 \lambda$. Em destaque os mineralóides de Calcita.

Outro fator importante é o elevado teor de $\mathrm{CaO}$ e $\mathrm{SiO}_{2}$, que foi mostrado na Tabela 1. Estes elementos são a base para a formação da Cuspidina ( $\left.\mathrm{Ca}_{4} \mathrm{Si}_{2} \mathrm{O}_{7} \mathrm{~F}\right)$, mineral 
do grupo dos sorosilicatos. Este composto não foi identificado no microscópio, mas sabe-se da sua existência já que o mesmo foi identificado através da análise por difração de Raios-X conforme pode ser visto na Figura 6 (a). Por fim, sabe-se que os fluxantes peritéticos devem possuir uma maior tendência a formar fases cristalinas quando comparado com fluxantes baixo carbono [1,7]. A figura 6 (b) mostra há formação de fases cristalinas no fluxante baixo carbono, mas estas fases formadas estão em menor quantidade e intensidade, portanto, não foi possível visualizar no microscópio, figura 3 (a).

Desta forma, a presente técnica aqui apresentada pode ser uma alternativa para fazer estudos comparativos com composições químicas variadas. Este tipo de análise pode viabilizar um estudo quantitativo de fases presentes e estas podem ser correlacionadas com as propriedades físicas do fluxantes em campo permitindo assim o desenvolvimento de composições que favoreçam um determinado tipo de aplicação específica.

(a)

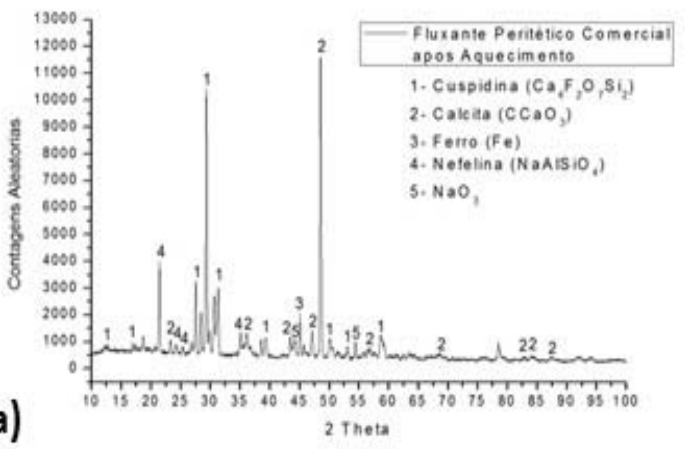

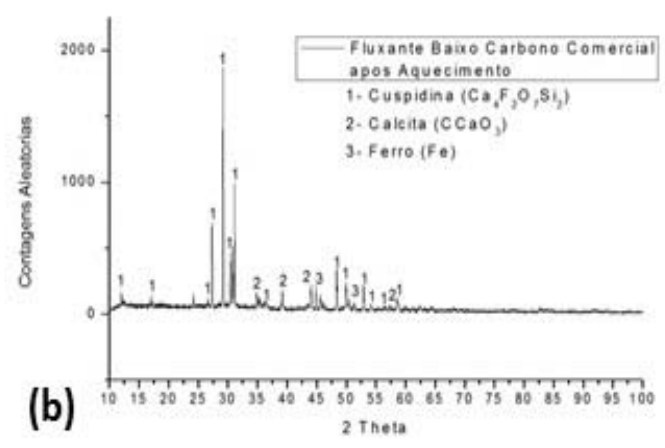

Figura 6. Análises de Raio-x após aquecimento do (a) Fluxante baixo carbono comercial, (b) Fluxante peritético.

\section{CONCLUSÃO}

A utilização de lâminas delgadas para caracterização de pós fluxantes, seguindo a metodologia utilizada na petrografia para descrição de minerais e rochas, mostra-se favorável e com resultados promissores.

Neste trabalho conseguiu-se diferenciar claramente dois tipos de fluxantes e foi observado a nítida formação de fase cristalina para o fluxante peritético e fase amorfa para o fluxante baixo carbono.

A fase cristalina presente observada na microestrutura é característica do mineralóide Calcita.

Não foi possível identificar a cuspidina na imagem metalográfica, mas sabe-se que esta está presente através das análises por difração de raios-X.

\section{Agradecimentos}

Os autores agradecem ao Instituto Federal de Ciência e Tecnologia do Espírito Santo - IFES pelo apoio e agradecem também pelo apoio financeiro: A FAPES Fundação de Amparo a Pesquisa do Espírito Santo; Ao CNPQ - Conselho Nacional de Desenvolvimento Científico e Tecnológico e a FINEP - Financiadora de Estudos e Projetos. À Fundação de Amparo à Pesquisa e Inovação do Espírito Santo FAPES por apoiar esta pesquisa. 


\section{REFERÊNCIAS}

[1] Li Z, Mills K, Bezerra MCC. Characteristics of mould flux films for casting MC and LC steels. XXXV Seminário de Fusão, Refino e Solidificação dos Metais. Salvador, Brasil. 2004.

[2] Garcia A, Spim JA, Santos CA, Cheung N. Lingotamento contínuo de aços. São Paulo: Associação Brasileira de Metalurgia e Materiais; 2006.

[3] Görnerup M, Hayashi M, Däcker CA, Seetharaman S. Mouldfluxes in continuouscastingofsteel - characterizationand performance tuning. VII International Conference on Molten Slags Fluxes and Salts, 2004.

[4] Mills KC, Fox AB, Thackray RP, LI Z. The performance and properties of mould fluxes. VII International on Molten Slags Fluxes and Salts. The South African Institute of Mining and Metallurgy. 2004.

[5] Cho JW, Emi T, Shibata H, Suzuki M. Heat transfer across mold flux film in mould during inicial solidification in continuous casting of steel. ISIJ International. 1998; 38(8): 834842.

[7] Li Z, Thackray R, Mills KC. A test to determine crystallinity of mould fluxes. VII International Conference on Molten Slags, Fluxes and Salts. The South African Institute of Mining and Metallurgy. 2004.

[8] Rodríguez P.; Arab P. B.; Celestino T. B.; Characterization of rock cracking patterns in diametral compression tests by acoustic emission and petrographic analysis. InternationJornal of Rock Mechanics Sciences. 73-85. 2016.

[9] Ribeiro, J.; Ruiz, S, I.; et al. Petrography and mineralogy of self-burning coal wastes from anthracite mining in the el bierzo coalfield (NW spain). International Journal of coal geology. 92-106. 2016.

[10] James R. C.; David J. V. Ore microscopyand ore petrography. Vaughan2 ed.1994.

[11] Cho JW, Emi T, Shibata H, Suzuki M. Heat transfer across mold flux film in mould during inicial solidification in continuous casting of steel. ISIJ International. 1998; 38(8): 834842.

[12] ASSOCIAÇÃO BRASILEIRA DE NORMAS TÉCNICAS, NBR 15845-1:2015: Rochas para revestimento, análise petrográfica, Rio de Janeiro: (1992)

[13] Cesero P. de. Mauro L. M..Ros L. F. Técnicas de Preparação de Lâminas Petrográficas e de Molde de Poros na Petrobras. Geoci. Petrobras, Rio de Janeiro, 105-116. 1989.

[14] Dana, E.S. \& HURLBUT, C.S. - Manual de Mineralogia. Editora Reverté, S.A. New York, USA, $2^{a}$ Edição, 1960. 600p.

[15] Richards, P. (1999) The Four Twin Laws of Calcite and How to Recognize Them. Rocks and Minerals: 74(5): 308-317.

[16] Skalwold, E.A. and Bassett, W.A. Double Trouble: Navigating Birefringence. Mineralogical Society of America, Chantilly, VA, 20 pages. ISBN 978-0-939950-027.2015 . 